\section{Untersuchungen zur Beeinflussung der Organlokalisierung von Influenza-Virus *}

Von

\section{H. FatrLaRd und H. StrotrL}

Physiologisch-Chemisches Institut (Direktor: Prof. Dr. Dr. W. KuENK)

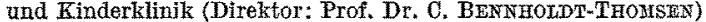
der Universität Köln

(Eingegangen am 20. Dezember 1961)

Ein großer Teil der Mikroorganismen besitzt die Fähigkeit, sich im Wirtsorganismus in bestimmten Organen und Geweben zu lokalisieren. Experimentelle Untersuchungen über die Verteilung des Influenza-Virus und seine mögliche Affinität zu versehiedenen Organen sind in vivo bisher kawm durchgeführt worden. Duroh radioaktive Markierung des Virus mit ${ }^{22} \mathrm{P}$ versuchten erstmals Iskrda, Kosaka und SASARI ${ }^{1}$ an Mäusen die Verteilung des Virus in versehiedenen Organen aufzuzeigen. Eine Anreicherung von Viren in Lungengewebe konnte bei einem Virus-Hämagglutinationstiter von 1024 bzw. 5120 Hämagglutinations-Dosen/ml exhalten werden (Messung der Radioaktivität im Liquid-Scintillation-Counter mit jeweils $0,1 \mathrm{ml}$ Virussuspension $+1 \mathrm{ml}$ Hyamine- 10 X Hydroxyd $+2 \mathrm{ml}$ Dioxan (mit 3,5 g PPO/1 (2,5-Diphenyloxazol) und $10 \mathrm{mg}$ POPOP/1 (1,4-bis-2-(5-Phenyloxazolyl-benzol)) + $3 \mathrm{ml}$ Tolnol (mit $4 \mathrm{~g}$ PPO und $100 \mathrm{mg}$ POPOP/1).

Applikation des Virus in zwei Versuchsreihen an je zwei mit Lungenmitochondrien sensibilisierte und je zwei normale Kaninchen durch intravenöse Injektion von jeweils $1 \mathrm{ml}$ radioaktiver Virussuspension entsprechend $1024 \mathrm{bzw} .5120 \mathrm{HD}$ mit $161000 \mathrm{bzw} .3900000 \mathrm{Imp} / \mathrm{min}$. Tötung der Tiere nach 4 Std (die sensibilisierten Tiere zeigten starken Anstieg der Körpertemperatur und Dyspnoe). Auszählen von je $10 \mathrm{mg}$ an verschiedenen Organbereichen entnommenem Organgewebe, nach vorangegangener 8-10stündiger Hydrolyse des Gewebes in jeweils $1 \mathrm{ml}$ Hyamine-10X Hydroxyd bei $40^{\circ} \mathrm{O}$, im Liquid-Scintillation-Counter nach Zusatz obengenannter Fhore in Dioxan-Toluol (Ergebnisse s. Tabelle).

Mit Lungenmitochondrien immunisierte Tiere $(1 \mathrm{a}-4 \mathrm{a})$ weisen eine eindeutig höhere Anreicherung von radioaktiv markiertem Virus im Lungengewebe auf gegenüber allen übrigen parenchymatösen Organen und gegenüber den Lungen nicht organsensibilisierter Tiere $(1 \mathrm{~b}-4 \mathrm{~b})$.

Verglichen mit den niedrigeren Serumwerten läßt der sehr hohe Anteil von Virus auf den Erythrocyten unter den hier angewandten. Versuchsbedingungen auf deren bekannte Transportfunktion für das Virus schließen, was auch in Übereinstimmung mit den Untersuchungen von HAMRE

u. Mitarb. über die experi-

Versuchsreihe 1 ausgeführt mit Virus vom Titer $1024 \mathrm{HD} / \mathrm{ml}$ und $154 \mathrm{Tmp} / \mathrm{HD}$.

Versuchsreihe 2 ausgeführt mit Virus vom Titer $5120 \mathrm{HD} / \mathrm{ml}$ und $761 \mathrm{Imp} / \mathrm{HD}$. innerhalb der ersten 3 Std nur nach intranasaler Installation und entsprechend im Gehirn nach intracerebraler und in der Leber intraperitonealer Applikation beobachtet werden. Eine spezifische Anreicherung von Influenza-Viren in bestimmten Organen, besonders in der Lunge, scheint demnach unter normalen Bedingungen nicht stattzufinden.

Da alle bisherigen Versuche keine gezielte Affinität der Viruspartikel zu den Zellen des Lungengewebes aufzeigen konnten, andererseits aber ein bevorzugter Befall der Respirationsorgane bei Influenzavirus-Erkrankungen bekannt ist, sollte hier untersucht werden, wie durch experimentelle Veränderungen an der Wirtszelle ein Organotropismus zustande kommen kann. Fine Möglichkeit hierzu bietet die Organsensibilisierung, die mit Mitochondrien als weitgehend organspezifischem Antigen in Anlehnung an KaBAT a. Mitarb. ${ }^{2}$ und HENLE $u$. Mitarb. ${ }^{3}$ durchgefïhrt wurde.

Als Versuchstiere dienten Kaninchen, da diese durch Influenzavirus nicht akut infiziert werden und somit die Ausbildung einer verstärkten Zellaffinität zum Virus besser in Erscheinung tritt.

Sensibilisiert wurden die Kaninchen durch intramuskuläre und subcutane Injektion von Kaninchen-Lungen-Mitochondrien, welche nach der Methode von SornEIDER w. HogEBOOM $^{4}$ isoliert wurden. Die Tiere erhielten im Abstand von jeweils 3-4 Wochen noch 4-6 Booster-Injektionen mit Mitochondrien- $\mathrm{Al}(\mathrm{OH})_{3}$-Suspensionen.

${ }^{32}$-radioaktiv markiertes Influenza-Virus wurde zur Vermeidung einer Verdünnung durch nicht-radioaktives Phosphat auf desembryonierten Hühnereiern in Anlehnung an die Vor-

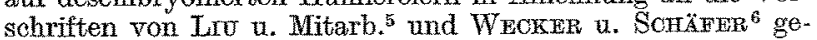
züchtet. Das Virus wurde durch mehrfache Ultrazentrifugation und gleichzeitiges Auswaschen mit der jeweils $10^{3}$-fachen Menge physiologischem $\mathrm{NaCl}$ gereinigt und angereichert. Nicht in die Viruspartikel eingebautes radioaktives Phosphat wurde hierdurch auf mindestens das $10^{\circ}$-fache verditinnt. Bei Einsatz von $200 \mu \mathrm{C}$ trägerfreiem Orthophosphat je Ei konnten Viruspräparate von $154 \mathrm{bzw}$. $761 \mathrm{Imp} / \mathrm{min} / \mathrm{Hämagglutinationsdosis}$

* Die Untersuchungen wurden durch Mittel der Deutschen Forschungsgemeinschaft unterstützt. mentelle Virämie der Maus steht. Die relativ hohe Virusanreichegans zurückzuführen sein und zusätzlich bei den organsensibilisierten Tieren noch auf einem unspezifisch bedingten, erhöhten Funktionszustand aktiver reticulärer Zellen beruhen.

Aus ähnlichen Gründen mag bei dem Versuchstier 4a, das ohnehin auffallend dystroph erschien, der hohe Impulswert im Lebergewebe mit nachträglich festgestellten, histologisoh nachweisbaren, entzündlich degenerativen Veränderungen des Parenchyms zusammenhängen.

Die großen Unterschiede der Virusanreicherung im Lungengewebe organsensibilisierter Kaninchen zu den Kontrolltieren, verglichen mit den relativ kleinen Differenzen beider Gruppen im Milzgewebe, lassen schließen, daß in diesem Falle die besondere Affinität des Lungenparenchyms zum Virus auf eine durch Lungenmitochondrien erzielte Organsensibilisierung zurüokzufübren ist. Bei primär für Influenza-Virus empfänglichen Mäusen hingegen werden die Unterschiede nicht in gleicher Art deutlich, worüber später ausführlicher berichtet werden soll.

Literatur. I ISHTDA, N., X. KOSAKA and H. SASAKT: Tôhoku J. exp. Med. 71, $163(1959) .{ }^{2}$ FuRTH, J., and M. A. KaBAT: J. exp. Med. 74, 247 (1941). $-{ }^{3}$ Hins. W, W., J. A. Cravbars and V. Groupé: J. infect. Dis. 74, 4.25 (1941). a Schnerdoz, W. C., and G. H. HoGeboom: Cancer Res. 11, $1(1951) .5$ Lrv, O. C., H. Brank, J. SpIzizen and W. HENLE: J. Immunol. 73, 415 (1954). - ${ }^{6}$ WECKER, E., u. W. SCHÄFER: Z. Naturforsch. 11 b, 181 (1956). - 7 HamRe, D., J. APPEI and G. Loosus: J. Lab. clin. Med. 47, 182 (1956).

Über die submikroskopisehen Einsehlufkörper bei Hepatitis Von

H. Braungmaner, K. Fellinger und F. Patasch Aus der

Medizinischen Univ.-Klinik in Wien (Vorstand: Prof. Dr. K. FELLINGER) (Eingegangen am 27. Dezember 1961)

Der von uns erstmalig 1957 erhobene Befund, daß es bei Serumhepatitis und Hepatitis epidemica zum Auftreten von 